\title{
Reflets
}

Revue ontaroise d'intervention sociale et communautaire

\section{L'Internet : nouvelle ressource ou nouvelle barrière?}

\section{Noël Thomas}

Volume 4, numéro 2, automne 1998

Personnes vivant avec une incapacité

URI : https://id.erudit.org/iderudit/026234ar

DOI : https://doi.org/10.7202/026234ar

Aller au sommaire du numéro

Éditeur(s)

Reflets : Revue ontaroise d'intervention sociale et communautaire

ISSN

1203-4576 (imprimé)

1712-8498 (numérique)

Découvrir la revue

Citer cet article

Thomas, N. (1998). L'Internet : nouvelle ressource ou nouvelle barrière? Reflets, 4(2), 195-200. https://doi.org/10.7202/026234ar

Tous droits réservés (C) Reflets : Revue ontaroise d'intervention sociale et communautaire, 1998
Ce document est protégé par la loi sur le droit d'auteur. L'utilisation des services d'Érudit (y compris la reproduction) est assujettie à sa politique d'utilisation que vous pouvez consulter en ligne.

https://apropos.erudit.org/fr/usagers/politique-dutilisation/ 


\title{
L'Internet : nouvelle ressource ou nouvelle barrière?
}

\author{
Noël Thomas ${ }^{1}$
}

\section{Introduction}

Les barrières physiques limitant l'accès aux édifices tombent graduellement et les personnes dont la mobilité est réduite s'en réjouissent. Les édifices publics et même privés sont rénovés. On ajoute des rampes d'accès, on enlève des marches et on agrandit les couloirs et les entrées. Ce qui est plus important encore, c'est que les attitudes ont changé. Il est présentement impensable pour un architecte de construire un édifice sans que ses plans ne prévoient des rampes d'accès pour les fauteuils roulants. Dans une ville, il est maintenant impensable de construire un trottoir que les personnes en fauteuil roulant ne peuvent utiliser. Tout n'est pas parfait mais les progrès sont évidents, et surtout appréciés!

Par ailleurs, il semble qu'on est encore à l'âge de pierre en ce qui concerne les nouvelles technologies de la communication et de l'information. Des millions de pages WEB ont été créées sur l'Internet sans considérer les conditions d'accès pour les personnes vivant avec un handicap. Sommes-nous à répéter les erreurs du passé? Faudra-t-il, comme pour les édifices, consacrer une décennie à rénover l'Internet pour le rendre accessible? Quand les conceptrices et concepteurs de sites auront-ils le réflexe de penser aux personnes qui ne peuvent voir, ne peuvent entendre, ne peuvent manipuler la souris, ne peuvent comprendre les sites complexes? 
Quelle est notre part de responsabilité, nous qui sommes spécialistes des relations humaines, des communications interpersonnelles et des professions dites aidantes dans cet état de fait? Que faire? Par où commencer?

\section{Les grands enjeux}

Évidemment, la situation est loin d'être simple. Au risque de généraliser, voici quelques éléments de la dynamique.

1) Le personnel technique. Les programmeurs, analystes et techniciens des nouvelles technologies sont généralement jeunes. Ils sont peu préoccupés par les problèmes d'accessibilité, car ils ne vivent pas personnellement avec un handicap. En particulier, la plupart des personnes qui développent des sites Internet sur le WEB ont une vision excellente, une motricité très développée, une orientation spatiale très raffinée et une compréhension rapide des choses techniques. C'est la génération "Nintendo», la génération qui n'a pas connu la vie sans ordinateur.

2) La clientèle. Les personnes vivant avec une incapacité ont fait des tentatives pour se tenir à la page et ont bénéficié des interfaces textuelles. Le bon vieux système d'opération DOS se prêtait particulièrement bien aux systèmes en braille. Mais voilà que le progrès apporte des systèmes graphiques qui, tout en faisant le bonheur de la plupart des utilisateurs, compliquent énormément la tâche d'une personne aveugle, par exemple.

Imaginez un instant que vous naviguez sur l'Internet.Votre écran d'ordinateur est fermé et une voix artificielle vous lit la page qui s'affiche. Que faire des nombreuses images, menus, liens hypertextes et des invitations à "cliquer ici»? Comment est-il possible de naviguer avec la souris quand on ne peut voir l'écran? Il faut constamment faire des mises à niveau. Ce sont des opérations particulièrement périlleuses qui nécessitent des efforts importants de formation. 
Plus que la formation, les coûts sont importants. En effet, il faut beaucoup d'argent à la clientèle qui doit s'équiper différemment à l'arrivée d'une nouvelle vague de technologies. Et il faut encore de l'argent pour la création et la mise à jour continuelle de sites Internet accessibles.

1) Des solutions divergentes. Il arrive souvent qu'un site WEB accessible à une clientèle, pénalise automatiquement une autre clientèle. Par exemple, pour les personnes dont la vision est réduite, l'utilisation de la voix, dans le genre "Real Audio», ajoute une composante fort importante, alors que c'est tout le contraire pour les malentendants. De la même façon, un site sans graphiques et sans cadres (en anglais «frames») est essentiel pour une personne qui ne peut voir, mais cela complique énormément la vie de personnes ayant des difficultés d'apprentissage. En somme, une bonne technique d'organisation de l'information à l'écran pour une clientèle est anathème pour une autre. Quel défi!

2) La problématique francophone. Pour la francophonie, la situation se complique du fait que la plupart des technologies sont développées en langue anglaise. Par exemple, les balayeurs (scanners) ne peuvent pas toujours lire les caractères accentués. De plus, le système de dictée nécessite l'achat d'une licence pour chaque langue utilisée. Même en anglais, les logiciels de dictée ne sont pas programmés pour comprendre «l'anglais avec un accent franco-ontarien» et le taux d'erreur augmente considérablement à moins de passer de longues heures à formater différemment la machine.

\section{Les clientèles et leurs besoins}

On peut regrouper de la façon suivante les clientèles ayant des difficultés d'accès.

1) Les personnes avec des handicaps visuels (cécité totale ou partielle, daltonisme). Leurs difficultés ont trait au fait de comprendre l'affichage à l'écran (lecture de textes, perception d'images, 
perception des couleurs, compréhension de tableaux), mais aussi à leur capacité d'interagir avec la souris.

2) Les personnes ayant des difficultés auditives. Leurs problèmes deviennent de plus en plus aigus avec l'arrivée du multimédia.

3) Les personnes ayant des difficultés de motricité. Par exemple, pensons aux personnes qui ne peuvent pas utiliser la souris ou encore, à celles qui tapent avec un bâtonnet.

4) Les personnes ayant des difficultés d'apprentissage. Pensons aux personnes ayant un retard intellectuel, à celles vivant avec la dyslexie ou ayant de la difficulté à percevoir des données complexes. Pour elles, il faut que l'information soit présentée de façon très simple, structurée et prévisible.

5) Les personnes ayant des difficultés en littératie. Pensons ici aux analphabètes, aux personnes qui n'ont pas d'habiletés informatiques ou encore à celles qui ne sont pas familières avec la langue utilisée par le site.

\section{Les gestes à poser}

De nombreuses solutions se dessinent graduellement à l'horizon et méritent réflexion.

1) Il y a certainement les considérations techniques. Relevant du domaine de l'ingénierie et de l'ergonomie des interfaces physiques, il s'agit de développer du matériel et des logiciels facilitant l'accessibilité. Encore mieux, il s'agit de voir à ce que les problèmes d'accessibilité fassent partie intégrante du développement et de la conception initiale de tous les nouveaux outils, quels qu'ils soient.

2) Il $y$ a des considérations conceptuelles et pédagogiques. Il faut penser à l'organisation de l'information, à son catalogage ainsi qu'aux principes et mécanismes d'interaction (par exemple, les implications de l'utilisation de formulaires).

3) Les considérations financières sont importantes. Elles font partie de la démarche de planification et de budgétisation. Par exemple, si on développe un site WEB ou encore si on développe un 
cours à distance, il faut penser au tout début aux clientèles ayant des difficultés d'accès. Il est toujours plus économique de planifier correctement au début que de corriger les erreurs plus tard.

\section{Le rôle des professionnels}

Les professionnels qui accompagnent les personnes ayant une incapacité doivent apporter leur soutien. Mais celui-ci sera de bien peu d'utilité, s'ils ne comprennent pas. Par conséquent, il faut aussi "se brancher», connaitre le potentiel des technologies, garder un esprit vivement critique, siéger sur des comités d'action, effectuer du lobbying politique et participer à toutes les activités encourageant l'accès universel.

Il faut d'abord parler à la clientèle pour bien connaître ses besoins et ses aspirations. Dans ce sens, un forum électronique bilingue sur l'Internet avait lieu du 19 au 30 octobre 1998. Il avait pour thème "Les nouvelles technologies d'apprentissage et les apprenants et les apprenantes ayant une incapacité». Organisé par le Network of Ontario Distance Educators (the NODE) et le Bureau des technologies d'apprentissage du gouvernement fédéral, ce forum a rejoint des francophones et des anglophones du Canada, des États-Unis, de la France et de l'Angleterre pour déterminer les grands enjeux, les rôles et les responsabilités de chacun en vue d'améliorer la situation actuelle. On peut d'ailleurs lire le contenu des échanges le site électronique suivant: http:// node.on.ca. On peut aussi entendre une entrevue en "Real Audio» avec une franco-ontarienne aveugle sur le site: http:// seb.village.ca:7070/ramgen/hburroug.rm. Ce forum a servi d'inspiration à la rédaction de cet article. 


\section{Note}

1. Noël Thomas est travailleur social professionnel. Depuis l'obtention de son M.S.W. en 1974, Noël a consacré une bonne partie de sa carrière à l'exploration et à la critique de l'utilisation des technologies dans les relations humaines. Il se spécialise présentement en travail, gestion et éducation à distance. On peut le rejoindre au courriel noel.thomas@village.ca. Cet article est aussi disponible sur l'Internet à http ://village.ca/publications/. 